\title{
Influência do método de preparo da casca do coco verde como biossorvente para aplicação na remoção de metais em soluções aquosas
}

\author{
Influence of method of preparation of coconut shell green as biosorbent \\ for application in removal of metals in aqueous solutions
}

\author{
Maria do Socorro Pinheiro da Silva | Giselle Santiago Cabral Raulino \\ Carla Bastos Vidal | Ari Clecius Alves de Lima | Ronaldo Ferreira do Nascimento
}

Data de entrada: 11/10/2012 | Data de aprovação: 23/04/2013

Resumo

As principais fontes de poluição por metais tóxicos são aquelas provenientes dos efluentes industriais, da mineração e das lavouras. A adsorção utilizando materiais de baixo custo, ou biossorventes, vem surgindo como uma alternativa economicamente atrativa para potenciais tratamentos na remoção e recuperação de metais tóxicos. Neste trabalho a influencia de diversos tratamentos na preparação do pó da casca do coco verde, empregado como material adsorvente, foram estudados (água fria, água quente, solução de albumina e solução de $\mathrm{NaOH}$ ) na remoção dos metais tóxicos $\mathrm{Cu}^{2+}, \mathrm{Cd}^{2+}, \mathrm{Ni}^{2+}, \mathrm{Pb}^{2+} \mathrm{e}$ $\mathrm{Zn}^{2+}$. Também foram analisados os parâmetros físico-químicos $\mathrm{pH}$, condutividade elétrica, cor e DQO nas "águas de lavagens" oriundas dos tratamentos efetuados no pó da casca do coco verde. Verificou-se que os tratamentos com $\mathrm{NaOH}$ e albumina obtiveram melhores resultados em termo de capacidades de adsorção para todos os metais estudados, respectivamente embora suas "águas de lavagens" terem apresentado características físico-químicas em maior desacordo com a legislação pertinente no estado do Ceará (PORTARIA DA SEMACE N. ${ }^{154 / 2002) ~ e m ~ r e l a c ̧ a ̃ o ~ a o ~ d e s c a r t e ~ d o s ~ m e s m o s, ~ c l a s s i f i c a d o s ~}$ como efluentes industriais.

Palavras-chave: Adsorção, metais tóxicos, bagaço da casca do coco verde.

\begin{abstract}
The main sources of pollution by toxic metals are those coming from industrial effluents, mining and farming. Adsorption using low cost materials, or biosorbents, is emerging as an economically attractive alternative treatment for the removal and potential recovery of toxic metals. In this study, the influence of various treatments using powder of green coconut shells was studied (cold water, hot water, albumin, sodum hydroxide) for removal of the toxic metals $\mathrm{Cu}^{2+}, \mathrm{Cd}^{2+}, \mathrm{Ni}^{2+}, \mathrm{Pb}^{2+}$ and $\mathrm{Zn}^{2+}$. We also analyzed physical and chemical parameters including $\mathrm{pH}$, electrical conductivity, color and COD in "washings" coming from the experiments. It was found that treatments with sodium hydroxide and albumin gave better results in terms of adsorption capacities for all metals studied, although the "washings" presented physicochemical characteristics in disagreement with the relevant legislation in the State of Ceará (SEMACE N.o 154/2002) on disposal of industrial effluents.
\end{abstract}

Key-words: Adsorption, toxic metals, green coconut shell powder. 


\section{Introdução}

Devido ao crescimento desordenado das cidades, várias atividades antrópicas, principalmente as industriais, têm consumido recursos naturais e gerado resíduos que contêm substâncias perigosas e tóxicas que afetam intensamente o meio ambiente (SINGHAL, MEHROTRA, 1991).

De acordo com a Organização dos Estados Americanos (OEA), as indústrias que mais contaminam o meio ambiente são as dos setores de mineração e metalurgia, que lançam diariamente grandes volumes de gases, resíduos aquosos ou sólidos, contendo elementos de toxicidade variada. (SINGHAL, MEHROTRA, 1991). Dentre esses resíduos gerados, destacam-se os metais tóxicos ou metais pesados. Tais espécies químicas são não degradáveis, podendo acumular-se nos componentes do ambiente onde manifestam sua toxicidade e são altamente móveis, tornando-se muito difícil acompanhar o destino destas espécies metálicas depois de introduzidas no ecossistema (BAIRD, 2002; TARLEY; ARRUDA, 2003a; AGUIAR PALERMO; NOVAES, 2002; PINO, 2005; SUD et al., 2008; VOLESKY, 2001)

A remoção destes poluentes provenientes de diversas fontes é realizada através de métodos convencionais de tratamentos físico-químicos, tais como: coagulação, floculação, precipitação, filtração, adsorção com carvão, ozonização e troca iônica. No entanto, tais métodos são bastante onerosos e envolvem longos períodos de detenção, o que dificulta sua implementação (BANDYOPADHYAY, BISWAS, 1998; BAILEY et al., 1998; GURGEL, 2007). Os processos que envolvem troca iônica e adsorção em carvão ativado, comumente utilizados por indústrias, são relativamente caros, pois envolvem alto custo de equipamento e operação (TARLEY, ARRUDA, 2003).

Diante das considerações mencionadas, fazem-se necessárias pesquisas por novas tecnologias envolvendo processos de biosorção, visando à redução de custos e aumentando a eficiência no tratamento.

Atualmente a utilização dos resíduos agroindustriais como adsorventes para a recuperação de efluentes industriais é uma prática viável (POLLARD et al., 1992). A literatura apresenta trabalhos com resíduos de cenoura; cascas de amendoim; arroz; nozes; bagaço de cana-de-açúcar, entre outros (NASERNEJAD et al., 2005; JOHNSON et al., 2002; SINGH et al., 2005; KIM et al., 2001; JUNIOR et al., 2007). O aumento do consumo de água do coco verde e a sua vocação natural para industrialização vêm causando problemas de disposição final do resíduo gerado, ou seja, as cascas dos frutos do coco são enviadas para lixões e aterros sanitários (ROSA et al., 2001). Assim, a utilização do pó da casca de coco verde como biossorvente de metais tóxicos, para o tratamento de efluentes, é uma forma de aproveitamento viável e útil tanto na reutilização de resíduos sólidos, já que os mesmos são sobras de processos produtivos e são encontrados em quantidades apreciáveis, quanto no tratamento de efluentes (SOUSA, 2007).

Normalmente, esses resíduos, têm origem regionalizada, resultando na minimização dos custos com transporte. Podem ainda ser reutilizados após os processos de adsorção, realizando a dessorção ou incineração tornando possível a recuperação do metal adsorvido.

É importante salientar, no entanto, que algumas vezes esses materiais necessitam de pré-tratamentos, com a finalidade de remover compostos fenólicos, por conferirem cor à água (BAILEY et al., 1998). Contudo a qualidade final das soluções resultantes desses tratamentos não são monitoradas, ou até mesmo tratadas.

Diante do cenário exposto, o presente trabalho teve como objetivo, avaliar a influencia de diversos tratamentos físico-químicos realizados na preparação do pó da casca do coco verde empregado como adsorvente na remoção de metais tóxicos, além de verificar a qualidade das soluções resultantes dos tratamentos empregados.

\section{Materiais e métodos}

\section{Reagentes e soluções}

Neste trabalho foram utilizadas soluções estoque de 100 mg. $L^{-1}$ de íons metálicos que foram preparadas a partir de seus respectivos sais: $\mathrm{Cu}\left(\mathrm{NO}_{3}\right)_{2} \cdot 6 \mathrm{H}_{2} \mathrm{O} \mathrm{Zn}\left(\mathrm{NO}_{3}\right)_{2} \cdot 6 \mathrm{H}_{2} \mathrm{O}, \mathrm{Cd}\left(\mathrm{NO}_{3}\right)_{2} \cdot 6 \mathrm{H}_{2} \mathrm{O}$, $\mathrm{Pb}\left(\mathrm{NO}_{3}\right)_{2}$ e $\mathrm{Ni}\left(\mathrm{NO}_{3}\right)_{2} \cdot 6 \mathrm{H}_{2} \mathrm{O}$ de grau analítico MERCK (São Paulo, Brasil).

\section{Obtenção do pó da casca de coco verde}

O pó da casca de coco verde (Cocos nucifera) foi fornecido pela Embrapa Agroindústria Tro- 
pical-CE (EMBRAPA/CE). O material foi obtido de acordo com as etapas: Trituração; Prensa hidráulica rotativa; Moinho e máquina classificadora. As cascas de coco verde foram passadas em um dilacerador (triturador com facas de corte e martelos desintegradores), equipamento semelhante ao utilizado na preparação de forragem para animais para obtenção das fibras. Em seguida as fibras obtidas foram prensadas em uma prensa "PRH" (prensa de rolos horizontais), para retirar o excesso de umidade e levadas a um moinho dotado de peneiras para obtenção de duas frações: pó e fibra. O pó obtido foi lavado e posto para secar à temperatura ambiente (Rosa et al., 2004; Carrijo et al., 2002) .A faixa granulométrica de 60-99 foi utilizada para o desenvolvimento do trabalho devido sua facilidade de obtenção e operação (SOUSA, 2007).

\section{Tratamentos físico-químicos}

Os tratamentos no pó da casca do coco verde foram executados, antes dos estudos de adsorção, com as seguintes soluções:

Água deionizada fria (temperatura ambiente) (T1),

Água deionizada na temperatura de $60^{\circ} \mathrm{C}$ (T2),

Albumina na concentração de $40 \mathrm{~g}$. L-1 (T3),

$\mathrm{NaOH}$ na concentração de $0,1 \mathrm{Mol} \cdot \mathrm{L}^{-1}$ (T4).

As amostras de pó foram postas em contato com as soluções de tratamento por 3 horas em béqueres de $500 \mathrm{ml}$, obedecendo à seguinte proporção: 20 g do pó (base seca) para $200 \mathrm{ml}$ de solução.

Após o período de 3 horas o pó foi filtrado e lavado com água destilada até que sua água de lavagem permanecesse com uma coloração o mais clara possível. As águas de lavagens foram guardadas, identificadas verificadas o volume das mesmas e feitas as seguintes análises físico-químicas: $\mathrm{pH}$ (pHmetro WTW Modelo: 3101i), condutividade elétrica (MICRONAL Modelo: B330), cor (Colorímetro DM-COR, DIGIMED, SÉRIE 42387) e DQO (bloco digestor Policontrol; colorímetro Thermo-Electron Corporation, Modelo: Dicolet Evolution 100). Os volumes de águas de lavagem utilizados foram medidos em béquer graduado de 4L (Nalgon). Após a lavagem o pó tratado foi seco em estufa a temperatura de $60^{\circ} \mathrm{C}$.

A "água de lavagem" refere-se à água utilizada no pré-tratamento do pó, ou seja sua limpeza inicial para remoção de compostos fenólicos, orgânicos, bem como na água utilizada após o tratamento a que o pó da casca do coco foi submetido. Dependendo do tipo de tratamento a que o pó da casca do coco verde é submetido a água de lavagem adquire características físicas e químicas bem diferenciadas. No caso do pó ser tratado com soluções ácidas ou básicas, a água de lavagem possuirá um caráter mais ácido ou básico devido à remoção do excesso destes (soluções ácidas ou básicas) no pó.

É de grande utilidade a caracterização de alguns parâmetros físico-químicos nesta "água de lavagem", uma vez que a mesma poderá se tornar um agente contaminador, dependendo de como e onde for executado o descarte da mesma.

\section{Efeito do tratamento físico-químico na capaci- dade de adsorção}

A fim de estimar a capacidade de adsorção do material adsorvente tratado, foram realizados experimentos em batelada em triplicata, onde 0,4 g do material tratado e $10 \mathrm{~mL}$ de solução sintética multielementar $\left(\mathrm{Pb}^{+2}, \mathrm{Ni}^{+2}, \mathrm{Cd}^{+2}, \mathrm{Zn}^{+2} \mathrm{e}\right.$ $\left.\mathrm{Cu}^{+2}\right)$ na concentração de $100 \mathrm{mg}$. $\mathrm{L}^{-1} \mathrm{em} \mathrm{pH}$ 5,0 foram mantidos sob agitação (175 rpm) à temperatura ambiente durante 3 horas (SOUSA, 2007). Então, foram filtradas em papel de filtro quantitativo (marca: QUANTY - faixa preta, diâmetro de $11 \mathrm{~cm}$ ). A determinação da concentração residual dos íons metálicos foi realizada utilizando-se um espectrofotômetro de absorção atômica (EAA - VARIAN Modelo: AA24OFS com sistemas de lâmpadas de catodo oco). A capacidade de adsorção do adsorvente, Q (mg do metal/ g do adsorvente) foi determinada com base na diferença de concentração dos íons metálicos usando a Equação 1.

$$
\mathrm{QW}=\mathrm{V}(\mathrm{Co}-\mathrm{Ce})
$$

onde,

Q é a capacidade de adsorção (mg. $\mathrm{g}^{-1}$ );

Co e Ce são as concentrações do soluto na solução inicial e em equilíbrio em (mg.L $\left.{ }^{-1}\right)$, respectivamente;

V é o volume da solução (L);

$\mathrm{W}$ a massa do adsorvente (g). 


\section{Teoria}

O processo de adsorção depende de vários fatores tais como: natureza do adsorvente, adsorvato e das condições operacionais. As características do adsorvente incluem: área superficial, tamanho do poro, densidade, grupos funcionais presentes na superfície e hidrofobicidade do material. Por outro lado, a natureza do adsorvato, depende: da polaridade, tamanho da molécula, solubilidade e acidez ou basicidade. As condições operacionais incluem, principalmente, temperatura, $\mathrm{pH}$ e natureza do solvente (RUTHVEN, 1984; COONEY, 1999). Interações entre a superfície do adsorvente e o adsorvato são resultados de forças atrativas entre essas espécies. De acordo com o tipo de forças envolvidas no processo de adsorção, podemos classificá-lo como adsorção física (fisissorção) e química (químissorção) (MOURA et al., 2011).

Na adsorção física nenhuma ligação é feita ou quebrada, sendo assim, a natureza do adsorvato permanece inalterada. Na adsorção química há a formação de ligações químicas entre o adsorvente e o adsorvato, e envolve o rearranjo de elétrons do fluido que interage com o sólido e a consequente formação da ligação química. $\mathrm{O}$ adsorvato sofre uma mudança química e é geralmente dissociado em fragmentos independentes, formando radicais e átomos ligados ao adsorvente. Em muitos casos, a adsorção é irreversível e é difícil separar o adsorvente do adsorvato (RUTHVEN, 1984).

\section{Resultados}

Na Tabela 1, encontram-se as capacidades de adsorção do pó da casca de coco verde submetido a diversos tratamentos físico-químicos e na Figura 1 podem ser observados os percentuais de remoção dos metais submetidos aos quatro tratamentos. No geral as amostras tratadas com albumina (T3) e $\mathrm{NaOH}$ (T4) obtiveram melhores resultados na remoção dos íons metálicos, ao passo que a amostra tratada com água quente apresentou os menores valores de capacidade de adsorção para todos os metais. Pode-se observar também que a capacidade de adsorção

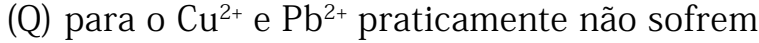
influência pelos diversos tratamentos, ao passo que zinco e níquel têm suas capacidades aumentadas quando submetidas aos tratamentos T3 e T4. O tratamento T4 foi o que apresentou melhor eficiência de remoção, segundo a Figura 1, para todos os metais estudados e menor efeito competição entre os metais e os sítios de adsorção. Sousa (2007) estudando a capacidade de adsorção do material bruto e tratado com NaOH 0,1 Mol.L-1 para os mesmos metais,

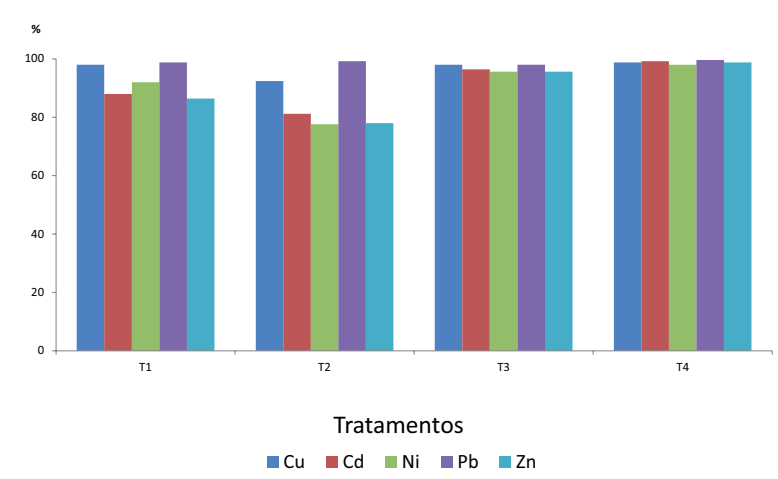

Figura 1- Eficiência de remoção dos metais submetidos aos tratamentos T1, T2, T3 e T4. Condições: $C_{0}: 100 \mathrm{mg} \cdot \mathrm{L}^{-1}$, massa do adsorvente: $0,4 \mathrm{~g}$, volume utilizado: $10 \mathrm{~mL}$, tempo de contato: 3 horas, Temperatura: $28 \pm 2{ }^{\circ} \mathrm{C}$.

observou também uma melhoria na capacidade de adsorção desse material.

O pó da casca de coco verde contém altos te-

\begin{tabular}{c|c|c|c|c|c}
\multicolumn{6}{c}{ CAPACIDADE DE ADSORÇÃO MÉDIA - QM (mg.g-1) } \\
\hline Tratamentos & \multicolumn{5}{c}{ Metais } \\
\hline & $\mathrm{Cu2}^{+}$ & $\mathrm{Cd2}^{+}$ & $\mathrm{Ni2}^{+}$ & $\mathrm{Pb2}^{+}$ & $\mathrm{Zn2}^{+}$ \\
\hline T1 & 2,45 & 2,20 & 2,30 & 2,47 & 2,16 \\
\hline T2 & 2,31 & 2,03 & 1,94 & 2,48 & 1,95 \\
\hline T3 & 2,45 & 2,41 & 2,39 & 2,45 & 2,39 \\
\hline T4 & 2,47 & 2,48 & 2,45 & 2,49 & 2,47
\end{tabular}

Tabela 1- Capacidade de adsorção média (QM) do pó da casca de coco verde submetido a diversos tratamentos físico-químicos. 
ores de lignina e celulose as quais possuem grupos hidroxila, metóxi e carboxílicos. Quando o material celulósico é submetido ao tratamento $\mathrm{T} 4$, os compostos fenólicos solúveis presentes na fibra celulósica são removidos e os sítios ativos ficam mais disponíveis, melhorando a adsorção (GURGEL, 2007; SALVADOR et al., 2009).

O tempo de contado requerido entre o adsorvente e o adsorvato para atingir a condição de equilíbrio é de fundamental importância para compreender os processos envolvidos durante a adsorção. Quando processos adsortivos ocorrem rapidamente, em sua grande maioria estes estão associados com o fenômeno de troca iônica, enquanto que, processos menos específicos, como por exemplo, os de complexação, os tempos envolvidos são consideravelmente maiores (TARLEY, ARRUDA, 2003). Neste trabalho, foi utilizado tempo de contato de 3 horas, pois é o tempo necessário para que ocorra o equilíbrio entre os metais e o biosorvente (Sousa, 2007).

Raulino (2011) utilizou o pó da casca do coco verde como adsorvente para remoção de metais tóxicos em coluna e observou que a utilização do pó da casca de coco verde tratado com $\mathrm{NaOH}$ 0,1 mol.L $\mathrm{L}^{-1}$ em coluna é viável na remoção dos metais cobre, níquel e zinco em soluções aquosas. Também é possível utilizar o pó da casca de coco verde sem tratamento para a remoção de metais, com a potencialidade de co-processamento desse material em outros setores industriais.

Na Tabela 2, encontram-se os resultados obtidos dos parâmetros analisados nas águas de lavagem de cada tratamento físico-químico realizado no pó da casca de coco verde. Pode-se observar pela Tabela 2 que o $\mathrm{pH}$ permanece na faixa da neutralidade. Para os tratamentos T1 a T3, esse resultado já era esperado já que a água e a solução de albumina não apresentam substâncias ácidas ou básicas em sua composição. Já no tratamento T4, o resultado obtido indica que houve reação entre a hidroxila presente em solução e o material lignocelulósico, acarretando num decréscimo do $\mathrm{pH}$ na água de lavagem final, além da diluição feita quando se lava o material com água após tratamento com hidróxido de sódio.

Quanto à cor, o tratamento T4 obteve o maior valor, comparado aos outros tratamentos. Mais um indicativo de que ocorreu reação entre o hidróxido de sódio e os compostos presentes no material lignocelulósico. A ação dos compostos alcalinos nesse tipo de material pode ocorrer através da desestruturação dos complexos lignocelulósicos, solubilizando a hemicelulose e expandindo a fração fibrosa, além de extrair os materiais orgânicos como os taninos (FILHO et al., 2003; ASADI et al., 2007). Como conseqüência, o volume gasto para lavagem do material submetido ao tratamento T4 foi maior que os demais, já que a cor liberada foi maior.

A "água de lavagem" oriunda do tratamento T3 apresentou maiores valores de demanda química de oxigênio (DQO) provavelmente devido à presença de uma proteína, a albumina, aumentando assim a carga orgânica. Nos tratamentos T2 e T4, os maiores valores de DQO em relação ao tratamento $\mathrm{T} 1$ se devem ao fato de a água quente e a solução de $\mathrm{NaOH}$ removerem compostos, como a lignina, a pectina, gorduras e taninos, que cobrem a superfície do material (GU, 2009; BRÍGIDA; ROSA, 2003).

A SEMACE (PORTARIA N. ${ }^{\circ} 154 / 2002$ ) e o CONAMA (PORTARIA 430/2011) estabelece valores para $\mathrm{pH}$ entre 5,0 e 9,0. A SEMACE

\begin{tabular}{c|c|c|c|c|c} 
Tratamentos & $\mathrm{pH}$ & $\begin{array}{l}\text { Condutivi- } \\
\text { dade } \\
(\text { Us/cm }\end{array}$ & $\begin{array}{l}\text { Cor } \\
(\mathrm{Pt}-\mathrm{Co})\end{array}$ & $\begin{array}{l}\text { DQO } \\
\left(\mathrm{mg} . \mathrm{L}^{-1}\right)\end{array}$ & $\begin{array}{l}\text { Volume } \\
(\mathrm{ml})\end{array}$ \\
\hline T1 & 5,75 & 503,0 & 545,0 & 130,62 & 1300,0 \\
\hline T2 & 5,62 & 604,0 & 803,0 & 291,61 & 1000,0 \\
\hline T3 & 6,26 & 2480,0 & 1340,0 & 5686,57 & 1350,0 \\
\hline T4 & 6,85 & 943,0 & 2580,0 & 383,33 & 1830,0 \\
\hline
\end{tabular}

Tabela 2- Resultados dos parâmetros físico-químicos efetuados nas "águas de lavagem" dos diversos tratamentos do bagaço. 
(PORTARIA N. $\left.{ }^{\circ} 154 / 2002\right)$ estabelece valores para DQO abaixo de 200mg. $\mathrm{L}^{-1}$ para descarte de efluentes industriais. Seria então necessário ainda um tratamento para redução de DQO da "água de lavagem" proveniente do tratamento T4 para que o mesmo pudesse ser descartado em um corpo hídrico. Considerando-se apenas o $\mathrm{pH}$ ambos estariam enquadrados para descarte. Ao compararmos os tratamento T1 e T4, considerando-se os parâmetros físico-químicos e as capacidades de adsorção (Tabelas 1 e 2 e Figura 1), foi possível notar que lavar o pó da casca de coco verde apenas com água pode ser vantajoso, pois utiliza-se menor volume de água na lavagem do material, menos cor é liberada assim como a DQO é menor. Além disso, não há diferença significativa na capacidade de adsorção dos dois tratamentos, indicando que o tratamento T1 é o mais adequado para o adsorvente, nesse estudo.

Raulino (2011) realizou estudo com o pó da casca do coco verde como adsorvente para remoção de metais tóxicos em coluna, realizando também teste de dessorção, a fim de avaliar a capacidade de reutilização do adsorvente, utilizando $\mathrm{HNO}_{3}$ 0,5 mol.L-1 como eluente e constatou que quase todo o cobre foi dessorvido (98,54\%), enquanto que apenas $50 \%$ do níquel e zinco foram dessorvidos.

Sousa et al (2010), estudando a remoção de metais utilizando o pó da casca de coco verde tratado com $\mathrm{NaOH} \mathrm{0,1}$ mol. $\mathrm{L}^{-1}$ verificou que o material adsorvente pode ser utilizado em mais um ciclo de adsorção, apesar de a partir do segundo ciclo ter sido observada a perda de eficiência na remoção dos metais.

A elevada eficiência de remoção dos metais tóxicos pelo pó da casca do coco verde mostra a potencialidade deste material. Ainda, por se tratar de um material em abundância e, devido suas características físicas como a de elevada porosidade, é perfeitamente viável efetuar o tratamento do efluente em colunas, ao invés dos processos em batelada sob agitação. Esta característica do tratamento torna-o bastante conveniente para a remoção dos metais em volumes elevados de efluente, num tempo relativamente curto. Como exemplo, pode-se tratar cerca de 1000L de um efluente similar ao utilizado neste trabalho empregando apenas $40 \mathrm{Kg}$ de adsorvente, com um custo muito reduzido, em um sistema de batelada. Se o sistema utili- zado for o de coluna, chega-se a tratar cerca de 5000L deste mesmo efluente, segundo dados de Raulino (2011).

Evidenciada a elevada capacidade de adsorção apresentada pelo resíduo industrial (pó da casca do coco verde), surge uma importante questão: o que fazer com o adsorvente contendo os metais tóxicos? O processo mais comumente empregado é sua disposição em aterros industriais, o que, além de ser um procedimento oneroso, não elimina o problema. Além desta alternativa, existe a possibilidade de incineração controlada deste material, visando à obtenção de uma cinza rica em óxido de metais, passível de ser reutilizada pelas indústrias metalúrgicas em novos processos. Ou ainda como fonte de energia na queima desse material em caldeira (com condições controladas) na própria indústria.

\section{Conclusões}

Os resultados indicaram que os tratamentos físico-químicos T4 e T3 (tratados com $\mathrm{NaOH}$ e albumina, respectivamente) obtiveram melhores resultados na capacidades de adsorção para todos os metais estudados, porém suas "águas de lavagens" apresentaram características físico-químicas, parâmetro DQO, em maior desacordo com a legislação pertinente no estado do Ceará (PORTARIA DA SEMACE N. $\left.{ }^{\circ} 154 / 2002\right)$ em relação ao descarte dos mesmos, classificados como efluentes industriais.

Sendo assim é mais adequado lavar o pó da casca de coco verde apenas com água (T1), pois utiliza-se menor volume de água na lavagem do material, menos cor é liberada e o valor da DQO é menor, além disso, não há diferença significativa na capacidade de adsorção dos metais quando se utiliza o adsorvente submetido ao tratamento $\mathrm{T} 1$ em relação aos outros tratamentos.

\section{Referências bibliográficas}

Aguiar Palermo, M. R. M.; Novaes, A. C. Remoção de metais pesados de efluentes industriais por aluminossilicatos. Química Nova, v. 25, p. 1145-1554, 2002.

Asadi, F.; Shariatmadari, H.; Mirghaffari, N. Modification of rice hull and sawdust sorptive characteristics for remove heavy metals from synthetic solutions and wastewater. Journal 
of Hazardous Materials, v. 154, p. $451-458$, 2007.

Bailey, S. E.; Olin, T. J.; Bricka, R. M.; Adrian, D. A review of potentially lowcost sorbents for heavy metals. Water Research, v. 33, p. 2469 2479, 1998.

Baird, C. Química Ambiental. Editora Bookman, Porto Alegre, 2002.

Bandyopadhyay, A.; Biswas, M. N. Removal of hexavalent chromium by synergism modified adsorption. Indian J. Environ. Pollut., v.18, N. 9, p. 662-671, 1998.

BRÍGIDA, A. I. S.; ROSA, M. F. Determinação do Teor de Taninos na Casca de Coco Verde (Cocos nucifera). Proc. Interamer. Soc. Trop. Hort. v. 47, p. 25-27, 2003.

Carrijo, O. A; Liz, R. S.; Makishima, N. Fibra da casca do coco verde como substrato agrícola. Horticultura Brasileira, v. 20, N. 4, p. 533-535, 2002.

CONSELHO NACIONAL DP MEIO AMBIENTE (CONAMA- PORTARIA 430/2011). http:// www.mma.gov.br/port/conama/legiabre. cfm?codlegi=646 - Acessado em 11 de outubro de 2012 .

COONEY, D.O. Adsorption Design for Wasterwater Treatment, Lewis Publishers, London, England, UK, 1999.

Filho, J. M. P.; Vieira, E. L.; Silva A. M. A.; Cezar, M. F.; Amorim, F. U. Efeito do Tratamento com Hidróxido de Sódio sobre a Fração Fibrosa, Digestibilidade e Tanino do Feno de Jurema-Preta (Mimosa tenuiflora. Wild). Revista Brasileira Zootecnia, v.32, n.1, p.70-76, 2003.

$\mathrm{Gu}, \mathrm{H}$. Tensile behaviours of the coir fibre and related composites after $\mathrm{NaOH}$ treatment. Materials and Design, v. 30, p. 3931-3934, 2009.

Gurgel, L. V. A. Mercerização e modificação química de celulose e bagaço de cana-de-açúcar com anidrido succínico e trietanolamina: Preparação de novos materiais quelantes para a adsorção de $\mathrm{Pb}$ (II), Cd (II), Cr (VI) e Cu (II). Dissertação de Mestrado, Universidade Federal de Ouro Preto, Brasil, 2007.

Johnson, P. D.; Watson, M. A.; Brown, J.; Jefcoat, I. A. Peanut hull pellets as a single use sorbent for the capture of $\mathrm{Cu}(\mathrm{II})$ from wastewater. Waste Management, v.22, p. 471-480, 2002.

Junior, O. K.; Gurgel, A. L. V.; Melo, J. C. P.; Botaro, V. R.; Sacramento, T. M. M.; Gil, R. P. F.; Frederic Gil, L. Adsorption of heavy metal ion from aqueous single metal solution by chemically modified sugarcane bagasse. Bioresource Technology, v. 98, p. 1291-1297, 2007.

Kim, J W.; Sohn, M. H.; Kim, D. S.; Sohn, S. M.; Know, Y. S. Production of granular activated carbon from waste walnut shell and its adsorption characteristics for $\mathrm{Cu} 2+$ ion. Journal Hazardous Materials, B85, p. 301-315, 2001.

MOURA, C.P., VIDAL, C.B., BARROS, A.L., COSTA, L.S., VASCONCELLOS, L.C.G., DIAS, F.S., NASCIMENTO, R.F. Adsorption of BTX (benzene, toluene, o-xylene, and pxylene) from aqueous solutions by modified periodic mesoporous organosilica. Journal of Colloid and Interface Science. 363, p. 626-634, 2011.

Nasernejad, B.; Zadeh, T. E.; Pour, B. B.; Bygi, M. E.; Zamani, A. Comparison for biosorption modeling of heavy metals (Cr (III), Cu (II), Zn (II)) adsorption from wastewater by carrot residues. Process Biochemistry, v. 40, p.1319-1322, 2005.

Pino, G. A. H. Biossorção de metais pesados utilizando pó da casca de coco verde (Cocos nucifera). Dissertação de Mestrado, Pontifícia Universidade Católica do Rio de Janeiro, Brasil, 2005.

Pollard, S. J. T.; Fowler, G. D.; Sollars. C. J.; Perry, R.. Low cost adsorbents for waste and wastewater treatment: a review. Sci. Total Environ., v.116, p.31-52, 1992. Pós - graduação em Química, 2006. Dissertação de Mestrado, 73p.

Rosa, M. F.; Abreu, F. A. P.; Furtado, A. A. L.; Brígido, A. K. L.; Norões, E. R. V. Processo agroindustrial: obtenção de pó de casca de coco verde. Fortaleza: Embrapa Agroindústria Tropical, $4 \mathrm{p}$ (Comunicado Técnico, 61), 2001.

Rosa, M. F.; Figueiredo, M. C. B.; Mattos, A. L. A.; Bezerra, F. C.; Crisostómo, L. A.; Araújo, A. M.; Abreu, F. A. P.; Veras, L. G. C.; Silva, J. T. Resúmenes de la 50a Reunión de la Sociedad Interamericana de Horticultura Tropical, La Mercedes de Guácimo, Costa Rica, 2004. 
RAULINO, G.S.C. Sistema piloto de adsorção de íons de metais em coluna (leito fixo) utilizando como adsorvente o pó da casca do coco verde. Dissertação de mestrado do curso de pós graduação em Engenharia Civil, aérea de concentração Saneamento Ambiental, Universidade Federal do Ceará, 2011.

RUTHVEN, D.M., 1984. Principles of Adsorption \& Adsorption Process. John Wiley \& Sons, New York.

Salvador, G.; Laus R.; Fávere, V. T. Adsorção de cobre(II) pela casca de coco verde condicionada com solução de $\mathrm{NaOH}$. XVII Encontro de Química da Região Sul (17SBQSul). Rio Grande do Sul, Brasil, 2009.

Singh, K. K; Hasan, S. H.; Rastogi, R. J. Hazard. Removal of cadmium from wastewater using agricultural waste rice polish. Journal of hazardous materials A121, p.51-58, 2005.

SINGHAL, R.K.; MEHROTRA, A.K. Process for the treatment of effluents on the mining. In: 2ND International Conference on Environmental Issues and Management of Waste in Energy And Mineral Production, may 30 - june 2,1992, Calgary Canada. Proceerf/ngs...Canada, 1991 p 1-12. solutions using sea nodule residue. Colloids and surfaces A, v. 237, p. 133-140, 2004.

SOUSA, F. W.; MOREIRA, S. A.; OLIVEIRA, A. G.; CAVALCANTE, R. M.; NASCIMENTO, R. F.; ROSA, M. F. Uso da casca de coco verde como adsorvente na remoção de metais tóxicos. Química Nova, v. 30, p. 1153 - 1157, 2007.

SOUSA, F. W.; OLIVEIRA, A. G.; RIBEIRO, J. P.; ROSA, F. M.; Keukeleire, D.; NASCIMENTO, R. F. Green coconut shells applied as adsorbent for removal of toxic metal ions using fixed-bed column technology. Journal of Environmental Management 91 pp. 1634-1640, 2010.

Sud, D.; Mahajan, G.; Kaur, M. P. Agricultural waste material as potential adsorbent for sequestering heavy metal ions from aqueous solutions - A review. Bioresource technology, v. 99, p. 6017 - 6027, 2008.

Superintendência Estadual do Meio Ambiente do Ceará (SEMACE - PORTARIA 154/2002). http://www.semace.ce.gov.br/biblioteca/legislacao/conteudo_legislacao.asp?cd=95 - Aces- sado em 26 de março de 2010.

Tarley, C. R. T.; Arruda, M. A. Z. Adsorventes naturais: potencialidade e aplicações da esponja natural (Luffa cylindrica) na remoção de chumbo em efluente de laboratório. Analytica, v. 2 , p. $25-31,2003$

Volesky, B. Detoxification of metal-bearing effluents: biosorption for the next century. Hydrometallurgy, v. 59, p. 203-216, 2001.

\section{SOBRE OS AUTORES}

Maria do Socorro Pinheiro da Silva (1)

Química Industrial. Mestranda em Química pela Universidade Federal do Ceará -UFC

\section{Giselle Santiago Cabral Raulino}

Química Industrial. Doutoranda em Engenharia Civil na área de concentração em Saneamento Ambiental pela Universidade Federal do Ceará -UFC

\section{Carla Bastos Vidal}

Tecnóloga em processos Químicos. Doutoranda em Engenharia Civil na área de concentração em Saneamento Ambiental pela Universidade Federal do Ceará -UFC

\section{Ari Clecius Alves de Lima}

Engenheiro Químico. Doutorando em Engenharia Civil na área de concentração em Saneamento Ambiental pela Universidade Federal do Ceará -UFC

\section{Ronaldo Ferreira do Nascimento}

Doutor em Química pela USP. Professor adjunto da Universidade Federal do Ceará -UFC

(1)Endereço para correspondência: Av. Humberto Monte s/n , Bl. 939 - Campus do Pici - Fortaleza - Ceará - CEP: 60455-970 - Brasil - Tel: +55 (85) 3366-9042 